\title{
Error Analysis and Accuracy Assessment of Mobile Laser Scanning System
}

\author{
Shouzhi $\mathrm{Xu}^{1, *}$, Pengfei Cheng ${ }^{2}$, Yu Zhang ${ }^{3}$ and Penghui Ding ${ }^{4}$ \\ ${ }^{I}$ School of Geodesy and Geomatics, Wuhan University, Wuhan, 430079, P.R. China \\ ${ }^{2}$ China National Quality Inspection and Testing Center for Surveying and Mapping Products, Beijing 100830, P.R. \\ China \\ ${ }^{3}$ Chinese Academy of Surveying and Mapping, Beijing 100830, P.R. China \\ ${ }^{4}$ National Calibration Center for Surveying Instrument, Beijing 100039, China. P.R. China
}

\begin{abstract}
Mobile Laser Scanning System (MLS) is integrated with GNSS, IMU, Laser Scanner and Digital Cameras, and other sensors taking vehicle as a carrier. It can quickly obtain spatial location and attribute information on both sides of roads. With the rapid development of mobile mapping technology, there is a great variety of MLSs. Therefore, how to evaluate the performance of these systems is getting more and more attention. In this paper, the error factors affecting the accuracy of MLS were analyzed. Based on these analyses, the accuracy assessment methods were proposed for three sensors and the system. The known baseline value was used to assess static positioning accuracy of GNSS, GT580 was used to assess attitude accuracy of IMU, total station was adopted to assess distance accuracy of Laser Scanner, and multi-tooth dividing table was used to assess angle measurement of Laser Scanner. Testing field was used to assess the plane and elevation accuracy of the system. The experiments show that the methods proposed in this paper are feasible even for cmlevel high precision of MLS. Therefore the research has practical significance and reference value.
\end{abstract}

Keywords: Accuracy assessment, Error analysis, GNSS, IMU, Laser Scanner, MLS.

\section{INTRODUCTION}

With the advent of information surveying, the way people gather information gradually developed from static collection with a single sensor to dynamic acquisition with multi-sensors. With the concepts being put forward such as "Smart City" and "Digital City", the demands for threedimensional spatial information are getting larger and larger in agriculture, transportation, communications, industrial, disaster monitoring and other fields, and the requirements of updating speeds for spatial information are getting higher and higher. Therefore, the traditional mapping methods are far from enough to meet the growing demands for spatial information due to the acquisition cost and quick updating speeds. Under this background, the Mobile Laser Scanning system came into being. MLS is integrated with Global Navigation Satellite System unit (GNSS), Inertial Measurement Unit (IMU), Laser Scanner and Digital Cameras and other advanced sensors in vehicle. The digital cameras are selectable. In the procedure of the vehicle, this system quickly collects a variety of information about the target area, such as spatial data, attribute data, real images and other information.

*Address correspondence to this author at the School of Geodesy and Geomatics, Wuhan University, Wuhan, Hubei, 430079, P.R. China;

Tel: 18210223990; E-mail: xsz2011@whu.edu.cn
In recent years, the research on MLS has been mainly centralized on three aspects: systems integration technology researches [1-6], system parameter calibration [7-9], and system applications [10-16]. With the development of MLS, more and more attention has been paid to its precision and the evaluation of its performance has also been increasingly getting important. However, literatures are rarely involved this field [17-19]. In this paper, we gave a comparatively overall accuracy assessment of MLS, including accuracy assessment of each sensor and the overall accuracy assessment of the system, and the steps of the corresponding assessment methods were put forward in detail. And then the feasibility and validity were demonstrated by an experiment.

\section{ERROR ANALYSIS OF MLS}

MLS is multi-discipline combination, multi-sensor integration and multi-data fusion system. At present, the mainstream MLSs at home and abroad are shown in Fig. (1). The working principle is that laser scanner is doing twodimensional scanning perpendicular to the travel direction, taking a vehicle traveling direction as the motiondimensional, and constituting three-dimensional scanning system.

At the same time, GNSS provides a vehicle's accurate location information, while IMU provides a vehicle's spatial attitude information. A three-dimensional point cloud data of 


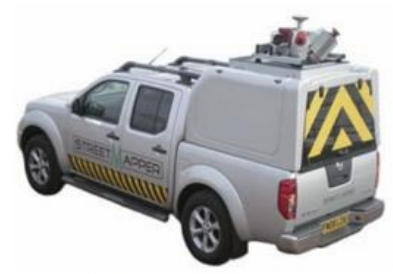

IGI StreetMapper

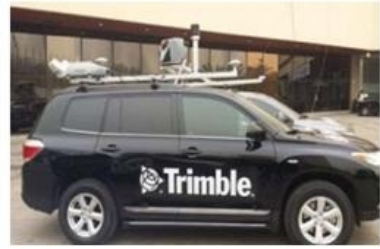

Trimble $\mathrm{MX8}$

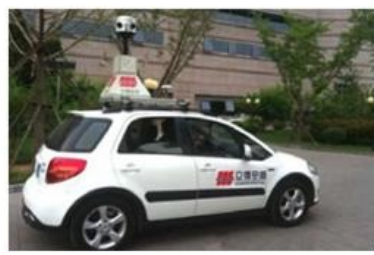

Leador MMS

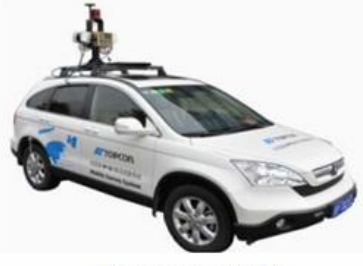

Topcon IP-S2

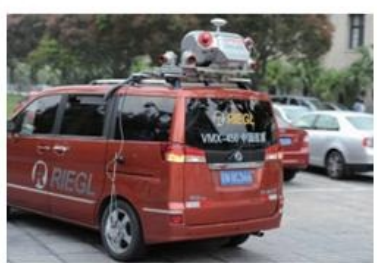

Riegl VMX-450

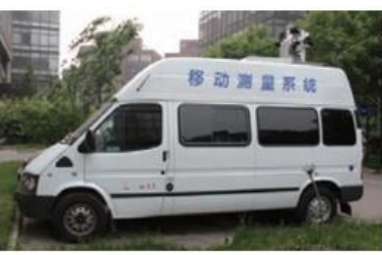

GEO-VISION SSW

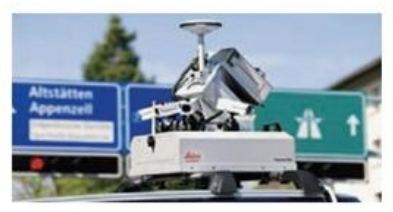

Leica Pegasus One

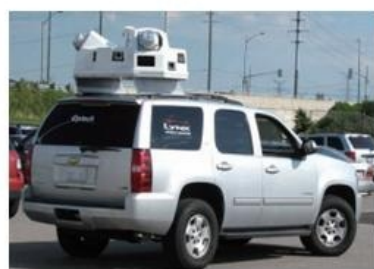

Optech LYNX

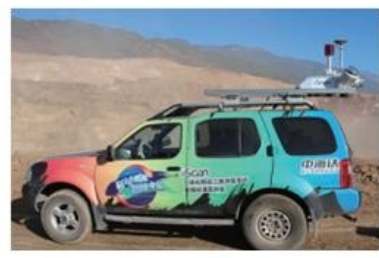

HiTarget iScan

Fig. (1). The mainstream MLSs at home and abroad.

scanned points are obtained through data fusion. Its positioning equation is shown as equation (1).

$$
\begin{aligned}
& {\left[\begin{array}{c}
x_{p} \\
y_{p} \\
z_{p}
\end{array}\right]=R_{L H}^{W}(t) R_{I M U}^{L H}(t)\left[k_{L}^{I M U} R_{L}^{I M U}\left[\begin{array}{l}
x_{L} \\
y_{L} \\
z_{L}
\end{array}\right]+\left[\begin{array}{c}
x_{L}^{I M U} \\
y_{L}^{I M U} \\
z_{L}^{I M U}
\end{array}\right]\right.} \\
& +\left[\begin{array}{c}
x_{L H}^{W}(t) \\
y_{L H}^{W}(t) \\
z_{L H}^{E}(t)
\end{array}\right]
\end{aligned}
$$

Where, $\left(x_{p}, y_{p}, z_{p}\right)$ is the scan point coordinates in the WGS-84 coordinate system, $R_{L H}^{W}(t)$ is the transformation matrix from local coordinate system to the WGS-84 coordinate system, $R_{I M U}^{L H}(t)$ is the transformation matrix from IMU coordinate system to local coordinate system, $k_{L}^{I M U}$ is the scale factor between laser scanner coordinate and IMU coordinate system, $R_{L}^{I M U}$ is the transformation matrix from laser scanner coordinate system to IMU coordinate system, $\left(x_{L}, y_{L}, z_{L}\right)$ is the scan point coordinates in laser scanner coordinate system, $\left(x_{L}^{I M U}, y_{L}^{I M U}, z_{L}^{I M U}\right)$ is the scanning center coordinates of laser scanner in IMU coordinate system, $\left(x_{L H}^{W}, y_{L H}^{W}, z_{L H}^{W}\right)$ is translation values between local coordinate system and WGS-84 coordinate system.
The MLS errors include the errors related with the sensors and system integration.

\subsection{Errors Related with the Sensors}

\section{1) GNSS errors}

According to the classification with error characteristics, there are stochastic errors and system errors. Stochastic errors include multi-path effects and observation errors, while systematic errors include orbit errors, satellite clock errors, receiver clock errors and atmospheric refraction errors. The corresponding error processing technology is relatively mature. The impacts of errors can be decreased or eliminated using the three techniques: model method, differential method and adjustment method. The model method can establish error correction model based on analysis of error characteristics, mechanisms and causes, or establish empirical fitting formula according to a large number of observed data. The differential method can eliminate or decrease its impact by differencing using the physical correlation of the errors between the observations. The adjustment method models error, introduces model parameters, and then put them together with other unknown parameters in the adjustment.

\section{2) IMU errors}

IMU errors mainly include component errors, installation errors, initial condition errors, principle errors, and outside interference errors. Component errors mainly refer to the gyro's drift and acceleration's zero bias and component's calibration error. Installation error refers to the error caused by accelerometer and gyroscope installed inaccurately. Initial condition error refers to the error formed by the inaccurate 
Table 1. The results of GNSS baseline calculated by post-processing software.

\begin{tabular}{|c|c|c|c|c|}
\hline Times & $\Delta \mathbf{X}(\mathbf{m})$ & $\Delta \mathbf{Y}(\mathbf{m})$ & $\Delta \mathbf{Z}(\mathbf{m})$ & Baseline values $(\mathbf{m})$ \\
\hline \hline 1 & 18775.2844 & 32417.2455 & 25056.0699 & 45068.7891 \\
\hline 2 & 18775.2809 & 32417.2472 & 25056.0697 & 45068.7887 \\
\hline 3 & 18775.2824 & 32417.2680 & 25056.0564 & 45068.7969 \\
\hline 4 & 18775.2824 & 32417.2508 & 25056.0646 & 45068.7891 \\
\hline
\end{tabular}

initial position and velocity input into IMU. Principle errors are caused by approximation error with mathematical models, the earth shape difference and gravity anomaly. Outside interference errors mainly refer to disturbing errors caused by vibration while the vehicle is moving.

3) Laser Scanner errors

Laser Scanner errors include ranging errors and angle measurement errors. Ranging errors are mainly classified into instrumental errors and environmental errors. Instrumental errors mainly refer to the time delay error problem in electro-optical circuit, which also includes a prism rotation error, vibration error, and the corresponding delay circuit errors. Environmental error is the ranging error caused by the different reflectivity due to the reflecting surface of the material, color, texture formation. The angle measurement errors with optical angular encoder mainly come from mechanical processing and installation. The mechanical processing and installation includes installation eccentric, installation tilt, rotation axis shaking and so on. The error caused by installation tilt and rotation axis shaking is very small and can be ignored.

\subsection{Errors Related with System Integration}

System integration errors include laser scanner's installation errors, time synchronization errors, and coordinate transformation errors and so on. (1) Installation errors. Each sensor has its own coordinate system, but sensors can not be guaranteed in accordance with the design attitude and position. Thus there are three boresight angles and lever arms. Installation errors cause systemic deviations in the results, so the three boresight angles and lever arms need to be determined firstly before the MLS measuring. (2) Time synchronization errors. The time reference of each different sensor has a certain difference, the respective time system needs to be unified into the UTC system. Moreover, the data interpolation will result in errors as the sampling frequencies are different in each sensor. (3) Coordinate transformation errors. The errors exist when coordinate transforming occurs due to the limitations of coordinate transformation model.

\section{ACCURACY ASSESSMENT OF MLS}

\subsection{Accuracy Assessment of Sensors}

1) Static accuracy assessment of GNSS.

GNSS provides location information for MLS. The following method was used to assess the location accuracy of
GNSS. The GNSS receiver was placed on known control point, and observed simultaneously with BJFS CORS station four times, and for an hour each time. The baseline value can be obtained using GNSS data post-processing software. The results are shown below in Table $\mathbf{1}$.

As the Table 1 showed, the average baseline value was $45068.79095 \mathrm{~m}$. Therefore the inner and external accuracy of GNSS can be calculated with the standard deviation and the root mean squared error (RMSE) respectively. The result showed that the inner accuracy was $3.97 \mathrm{~mm}$ and the external accuracy was $4.79 \mathrm{~mm}$.

2) Accuracy Assessment of IMU.

IMU provides attitude information for MLS. The IMU accuracy evaluation mainly includes the accuracy of roll angle, pitch angle, heading angle, and zero drift. The high accuracy reference is provided by GT580 dual-axis digital display type manual turntable. The turntable is composed of two parts, body and digital display table. The turntable's body is composed of inner ring shaft axis and the outer axis. The inner is an axis bearing of $360^{\circ}$, and its accuracy is $\pm 5^{\prime \prime}$, the accuracy of outer shaft is about \pm 7 " with a resolution of $1 "$.

The turntable's performance is shown in Table $\mathbf{2}$, and its appearance is shown in Fig. (2).

The accuracy evaluation methods of roll angle include three major steps in our study. The first step is leveling the turntable precisely using level tube before installing IMU on the leveling turntable, and then booting initialization for 15 minutes. The second step is adjusting the turntable and IMU on the same axis to ensure the change of pitching angles is very small when the roll angle is turned. The last step is setting digital display table zero, and then recording the IMU observed value $\left(\alpha_{\mathrm{i}}\right)$ and the results $\left(\varphi_{\mathrm{i}}\right)$ shown in data display instrument when rotating turntable every $10^{\circ}$.

The observed values of IMU roll angle are shown in Table 3. And the accuracy of roll angle $\left(\sigma_{\varphi}\right)$ is calculated and shown as $\sigma_{\varphi}=\sqrt{\frac{\sum_{i=1}^{n} v_{i}^{2}}{n-1}}=0.070^{\circ}$.

The accuracy assessment methods for pitch angle and heading angle are in the same way as the accuracy evaluation with the roll angle. The accuracy of pitch angle $\left(\sigma_{\theta}\right)$ was equal to $0.073^{\circ}$, and the accuracy of heading angle $\left(\sigma_{\Psi}\right)$ was equal to $0.043^{\circ}$. 


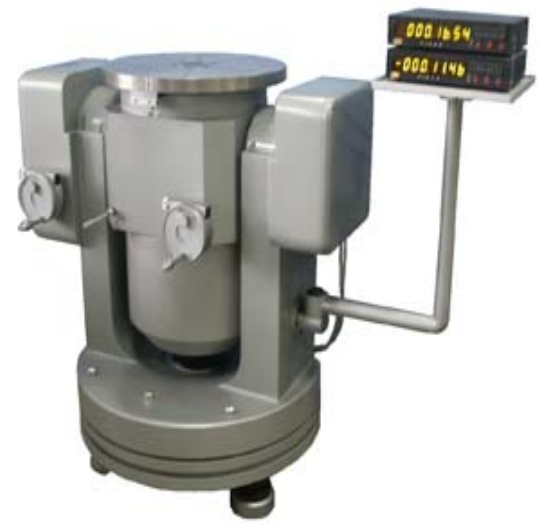

Fig. (2). GT580 dual-axis digital display turntable.

Table 2. The performance of GT580 biaxial digital manual turntable.

\begin{tabular}{|c|c|c|}
\hline Performance Indicators & Outer Axis (pitch) & Inner Shaft (Orientation) \\
\hline \hline Rotation range & $-92^{\circ} \sim+92^{\circ}$ & $-360^{\circ} \sim+360^{\circ}$ \\
\hline Angled rotation error & $\pm 3^{\prime \prime}$ & $\pm 2^{\prime \prime}$ \\
\hline Locating accuracy of angular positions & $\pm 5^{\prime \prime} / \pm 7^{\prime \prime}$ & $\pm 3^{\prime \prime} / \pm 5^{\prime \prime}$ \\
\hline Digital display measuring resolution & $11^{\prime \prime}$ & $1^{\prime \prime}$ \\
\hline
\end{tabular}

Zero drift refers to the dispersion degree around the mean of the output of the fiber optic gyroscope in a stationary state. It express as angle rate which is equivalent to the standard deviation of the output of IMU. The formula is following as equation (2).

$B_{s}=\frac{1}{k}\left[\frac{1}{n-1} \sum_{i=1}^{n}\left(F_{i}-\bar{F}\right)^{2}\right]^{1 / 2}$

Where: $B_{s}$ is zero drift, $F_{i} F_{i}$ is output of IMU, $\bar{F}$ is the mean of output, $\mathrm{n}$ is the number of samples.

As the key performance indicators of IMU, zero drift needs to be assessed for its accuracy. In this paper, attitude angle data of IMU was one hour static collection, its output frequency was 1Hz; the output data is shown in Fig. (3).

As seen from Fig. (3), the bias of roll and pitch is only 0.05 degree, but the bias of heading is 3 degree. It shows that the heading stability is very bad. Because the heading is related to the real north, it needs to initialize for low accuracy IMU. And the Zero drift accuracy assessment is computed by equation (2). Zero drift of roll is 0.011 , Zero drift of pitch is 0.009 , and Zero drift of heading is 0.706 .

\section{3) Accuracy assessment of Laser Scanner}

The laser scanners of MLS are usually two-dimensional laser scanners, and its working principle is that the laser pulse is emitted by the diode laser pulse. It scans the target point by rotating prism, and then receives and records the reflected laser pulse through the detector thereby acquiring the three-dimensional coordinates of the target point. The measurement principle is to measure the distance by the propagation time or phase change, and to obtain the angle value of the laser beam within the instrument by a precision clock control encoder. The origin point of the laser scanner coordinate system is a laser emitting a reference, $\mathrm{x}$-axis direction point is the vehicle direction, $\mathrm{z}$-axis direction perpendicular to the direction of vehicle direction, and y-axis is to meet the right hand rule. Therefore, the laser spot coordinate at the foot of the laser scanner $\mathrm{P}\left(x_{L}, y_{L}, z_{L}\right)$ is shown as equation (3). Where, $\theta$ is a scanner angle, $S$ is a scanner distance.

$\left[\begin{array}{l}x_{L} \\ y_{L} \\ z_{L}\end{array}\right]=\left[\begin{array}{l}0 \\ S \sin \theta \\ S \cos \theta\end{array}\right]$

Accuracy assessment of laser scanner includes measuring angle and range accuracy.

a) Range accuracy of Laser Scanner

The standard values were obtained by total station Sokkia NET05; its precision is $(1+1 \mathrm{ppm} * \mathrm{D}) \mathrm{mm}$. The range accuracy was computed using the observed value of the laser scanner to minus the standard values by root-mean-square error. The range data observed is shown in Table 4.

b) Angle measuring accuracy of Laser Scanner

Laser scanner accuracy was assessed using multi-tooth dividing table. It referenced the comparative method that the standard deviation test method round horizontally with a total 


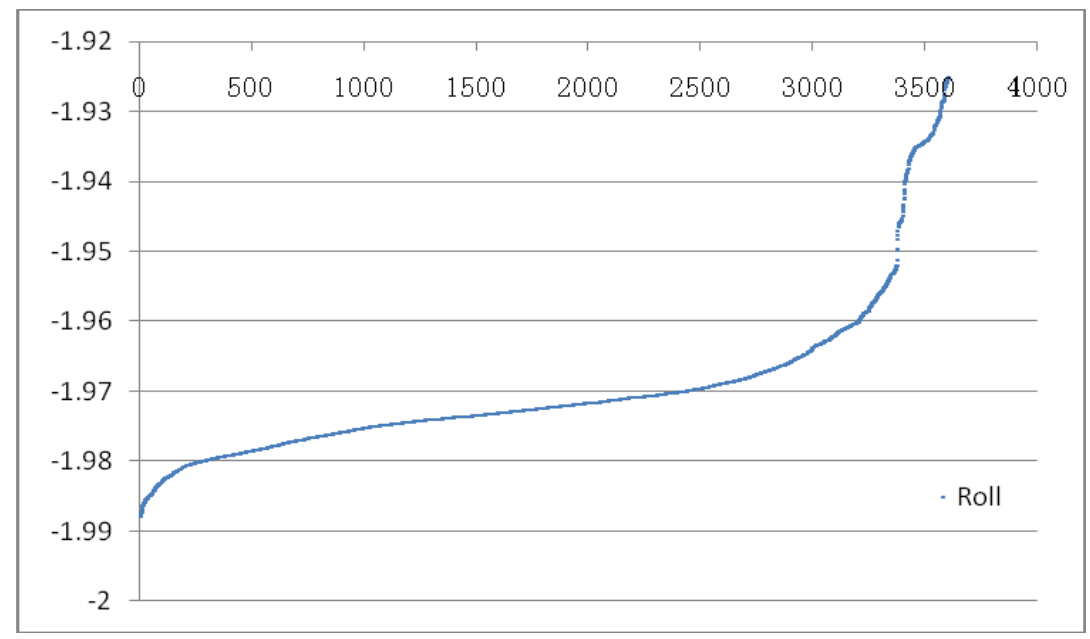

(a). Roll output

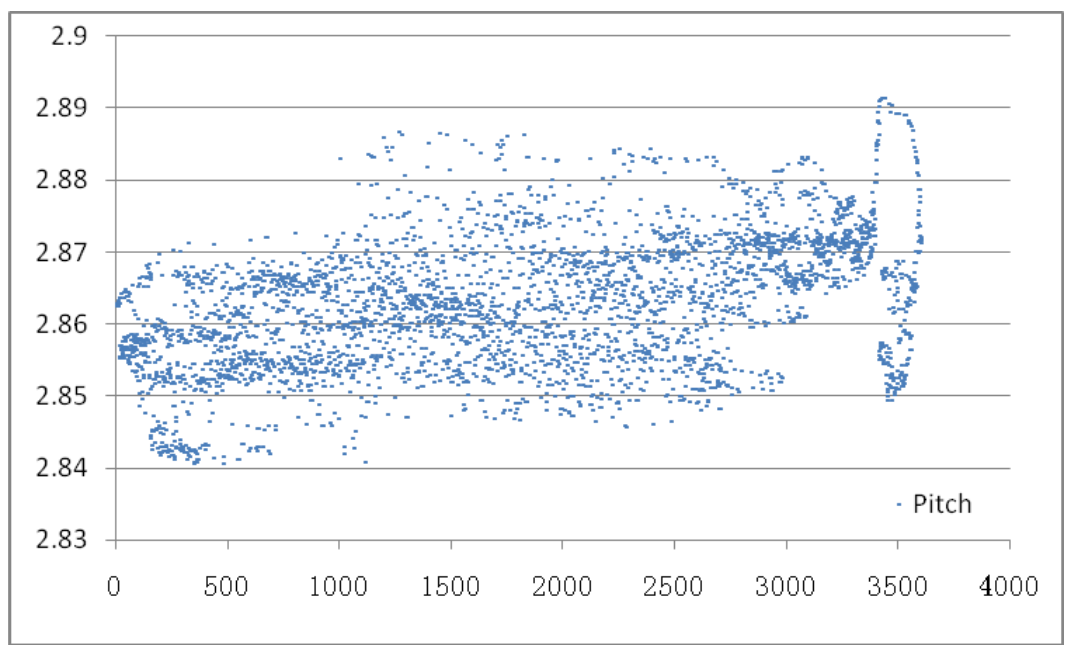

(b). Pitch output

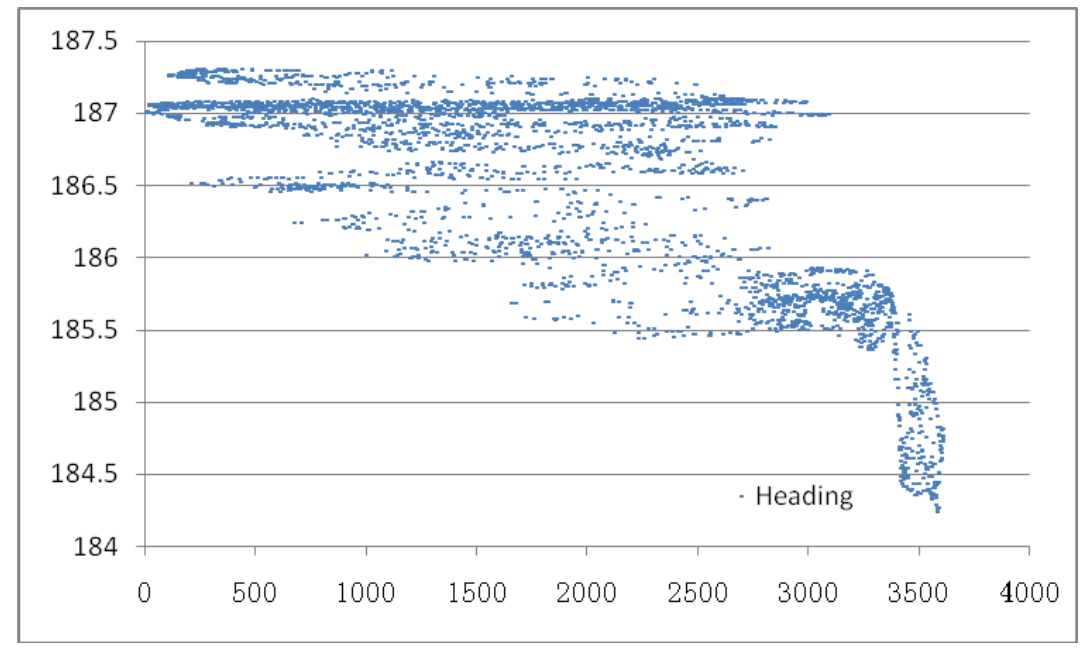

(c). Heading output

Fig. (3). The output of IMU attitude in an hour. 
Table 3. The observed value of IMU roll angle in degrees.

\begin{tabular}{|c|c|c|c|c|c|}
\hline Id & Observed Value & Turntable Value & Angle of Observed Value & Angle of Turntable & Residual Error(vi) \\
\hline 1 & -0.0194 & 0.0000 & & & \\
\hline 2 & 10.1858 & 10.1236 & 10.2052 & 10.1236 & 0.082 \\
\hline 3 & 20.2227 & 20.0978 & 10.0369 & 9.9742 & 0.063 \\
\hline 4 & 30.3354 & 30.1564 & 10.1127 & 10.0586 & 0.054 \\
\hline 5 & 40.5508 & 40.2772 & 10.2154 & 10.1208 & 0.095 \\
\hline 6 & 50.6237 & 50.2764 & 10.0729 & 9.9992 & 0.074 \\
\hline 7 & 40.3512 & 40.0633 & -10.2725 & -10.2131 & -0.059 \\
\hline 8 & 30.1418 & 29.9214 & -10.2094 & -10.1419 & -0.067 \\
\hline 9 & 19.9571 & 19.8581 & -10.1847 & -10.0633 & -0.121 \\
\hline 10 & 9.9194 & 9.8997 & -10.0377 & -9.9583 & -0.079 \\
\hline 11 & -0.1809 & -0.1689 & -10.1003 & -10.0686 & -0.032 \\
\hline 12 & -11.0415 & -10.9775 & -10.8606 & -10.8086 & -0.052 \\
\hline 13 & -20.1801 & -20.0625 & -9.1386 & -9.0850 & -0.054 \\
\hline 14 & -30.3947 & -30.2247 & -10.2146 & -10.1622 & -0.052 \\
\hline 15 & -40.3832 & -40.1536 & -9.9885 & -9.9289 & -0.060 \\
\hline 16 & -50.3908 & -50.1119 & -10.0076 & -9.9583 & -0.049 \\
\hline 17 & -40.2721 & -40.0647 & 10.1187 & 10.0472 & 0.071 \\
\hline 18 & -30.0485 & -29.8875 & 10.2236 & 10.1772 & 0.046 \\
\hline 19 & -20.1207 & -20.0144 & 9.9278 & 9.8731 & 0.055 \\
\hline 20 & -9.9733 & -9.9547 & 10.1474 & 10.0597 & 0.088 \\
\hline 21 & -0.0418 & -0.0767 & 9.9315 & 9.8781 & 0.053 \\
\hline
\end{tabular}

Table 4. Laser scanner distance observation in meters.

\begin{tabular}{|c|c|c|c|}
\hline Distance & Laser Scanner & Total Station & Error \\
\hline \hline \multirow{3}{*}{10} & 9.950 & 9.959 & -0.009 \\
\cline { 2 - 5 } & 9.956 & 9.959 & -0.003 \\
\cline { 2 - 5 } & 9.960 & 9.959 & 0.001 \\
\cline { 2 - 5 } & 9.960 & 9.959 & 0.001 \\
\cline { 2 - 5 } & 9.954 & 9.959 & -0.005 \\
\cline { 2 - 5 } & 19.324 & 19.319 & 0.005 \\
\cline { 2 - 5 } & 19.352 & 19.319 & 0.033 \\
\cline { 2 - 5 } & 19.324 & 19.319 & 0.005 \\
\cline { 2 - 5 } & 19.326 & 19.319 & 0.007 \\
\hline
\end{tabular}


Table 4. contd..

\begin{tabular}{|c|c|c|c|}
\hline \multirow{2}{*}{ Distance } & Laser Scanner & Total Station & Error \\
\hline \hline \multirow{5}{*}{50} & 49.750 & 49.779 & -0.029 \\
\cline { 2 - 5 } & 49.764 & 49.779 & -0.015 \\
\cline { 2 - 5 } & 49.738 & 49.779 & -0.041 \\
\cline { 2 - 5 } & 49.770 & 49.779 & -0.009 \\
\cline { 2 - 5 } & 49.750 & 49.779 & -0.029 \\
\hline
\end{tabular}

The range accuracy is: $0.005 \mathrm{~m} @ 10 \mathrm{~m}, 0.016 \mathrm{~m} @ 20 \mathrm{~m}, 0.027 \mathrm{~m} @ 50 \mathrm{~m}$.

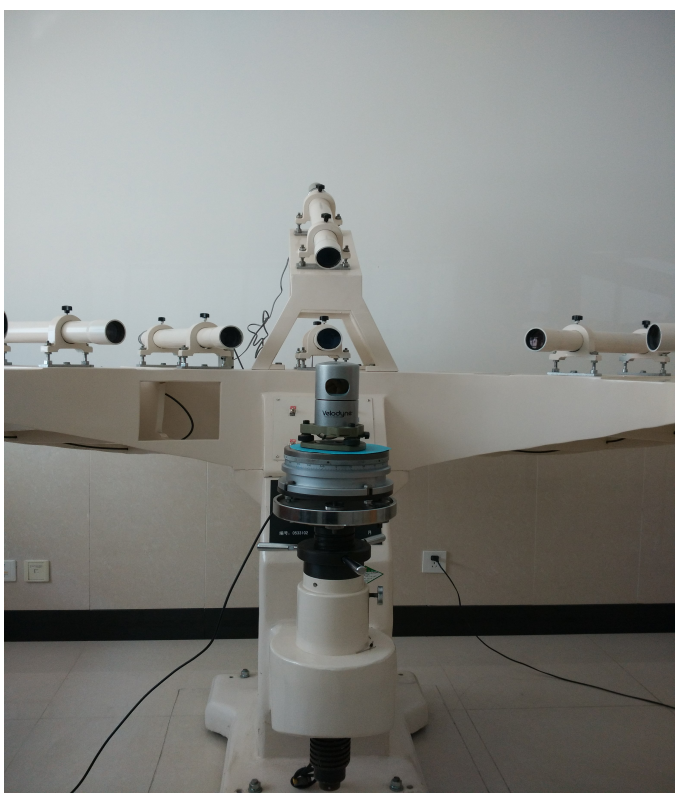

Fig. (4). Multi-tooth dividing table.

station and electronic theodolite, that is, the multi-tooth dividing table was taken as an angle of the standard device, and compared with the observed values obtained by laser scanner.

Multi-tooth dividing table is shown in Fig. (4) that is a kind of circular dividing standard instrument with high accuracy. Generally, it consists of two toothed plates with the same tooth and modulus. When such table is used, a tooth plate is fixed and is off with another. It meshes when rotating to the desired angle, so as to obtain positioning and high degree. Multi-tooth dividing table in 552 was used, and the maximum error is about $0.3^{\prime \prime}$.

Angular accuracy assessment methods contain two phases. The first phase was to level precisely the multi-tooth dividing table, fix the laser scanner to the platform and then level it together with foundation (Fig. 4). The second phase was to set the multi-tooth dividing table zero, and rotate clockwise with a certain angle each time. And then the scanner also followed the same rotating angle. The scanner scanned the target when the rotation finishes, and the corresponding angle value was extracted from the target. The laser scanner angle value and its accuracy are shown in Table 5.

\subsection{Evaluation of the System Accuracy}

The final data processed from MLS are threedimensional coordinates of point clouds. Therefore, the coordinate precision of point clouds is an important indicator of the system performance evaluation. Coordinate precision of point clouds includes plane precision and elevation accuracy. The accuracy assessment method is as follows: the feature points on both sides of the road, whose threedimensional coordinates can be obtained by the traditional way with high precision, can be regarded as the known points and their three-dimensional coordinates as the standard values. And then the system accuracy can be evaluated by comparing the results obtained from MLS with the standard values of the known feature points.

System accuracy assessment was carried out in the testing field (Fig. 5), which was $1000 \mathrm{~m}$ long from east to west and $550 \mathrm{~m}$ wide from north to south. The testing field was composed of the 45 known control points measured by RTK (with an expected accuracy of $1 \mathrm{~cm}+2 \mathrm{ppm}$ in horizontal plane and $2 \mathrm{~cm}+2 \mathrm{ppm}$ in height) and 200 measured points by total stations (Sokkia NET05). 


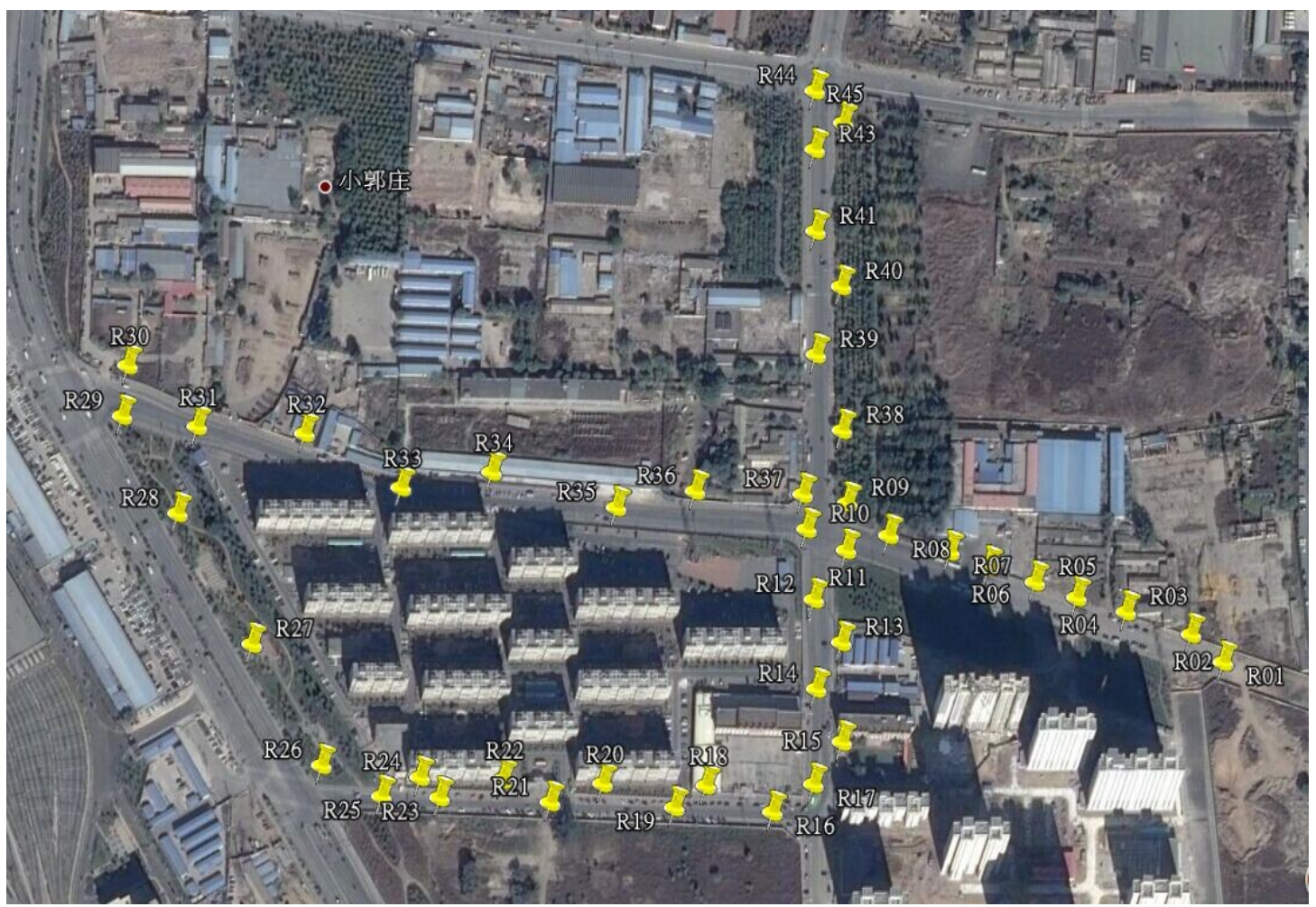

Fig. (5). The testing field.

Accuracy assessment procedure mainly included four steps. The first step was to combine GNSS data and IMU data by integrated navigation software in order to obtain MLS vehicle track. The second step was to preprocess laser scanner data using laser pretreatment software. The third step was to fuse track data and laser data for obtaining WGS-84 coordinates of point clouds by using point cloud processing software. The last step was to extract coordinates of feature points (such as: building corner, corner windows, poles, traffic signs, etc.) in the testing field, and compare them with the known points, and then assess their accuracy with equation (4).

$$
\left\{\begin{array}{l}
\sigma_{x}= \pm \sqrt{\frac{\sum_{i=1}^{n}\left(x_{p}-x_{r}\right)^{2}}{n}} \\
\sigma_{y}= \pm \sqrt{\frac{\sum_{i=1}^{n}\left(y_{p}-y_{r}\right)^{2}}{n}} \\
\sigma_{h}= \pm \sqrt{\frac{\sum_{i=1}^{n}\left(h_{p}-h_{r}\right)^{2}}{n}} \\
\sigma_{p}=\sqrt{\sigma_{x}^{2}+\sigma_{y}^{2}}
\end{array}\right.
$$

Where, $\left(x_{p}, y_{p}, z_{p}\right)$ is a point coordinate which is computed by MLS, $\left(x_{r}, y_{r}, z_{r}\right)$ is the coordinate of the reference point, $\sigma_{p}$ is the plane accuracy, $\sigma_{h}$ is elevation accuracy.

Plane error and elevation error of MLS are shown in Fig. (6) and Fig. (7). We selected nearly more than 90 obvious feature points in the testing field to assess accuracy, and the plane accuracy was $0.187 \mathrm{~m}$ and elevation accuracy was $0.251 \mathrm{~m}$ according to equation (4).

\section{DISCUSSION}

The accuracy of MLS is related to the accuracy of each sensor besides the level arm and boresight angle. The level arm refers to the distance from the center of the GNSS coordinate system to the center of the IMU coordinate system, and the distance from the center of the Laser Scanner coordinate system to the center of the IMU coordinate system. The boresight angel refers to the angel between laser scanner coordinate system to IMU coordinate system. Therefore, it requires precise calibration of level arm and boresight angle before accuracy assessment.

GNSS unit of MLS is a dynamic surveying process. But we assessed the static surveying accuracy of GNSS, because it is hard to assess the dynamic surveying accuracy, it 


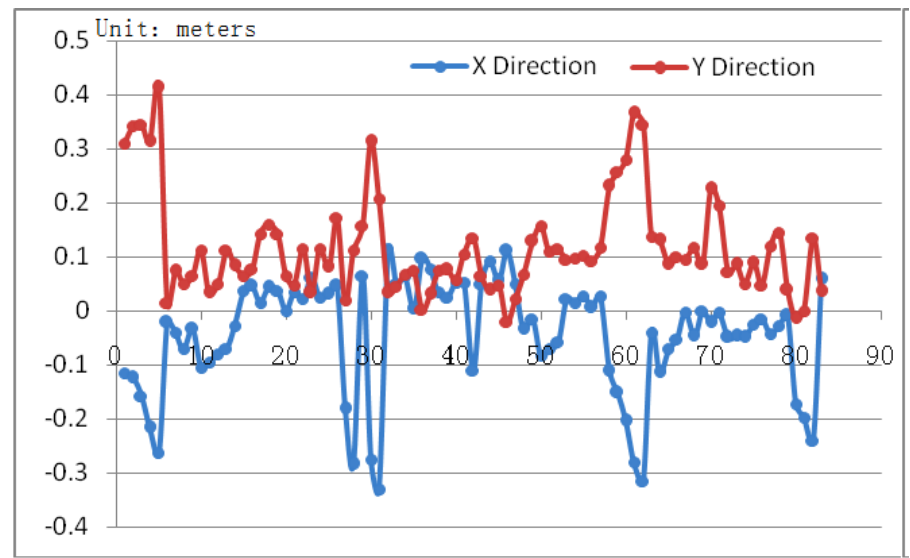

Fig. (6). The differential value of coordinates in $\mathrm{x}$ direction and $\mathrm{y}$ direction.

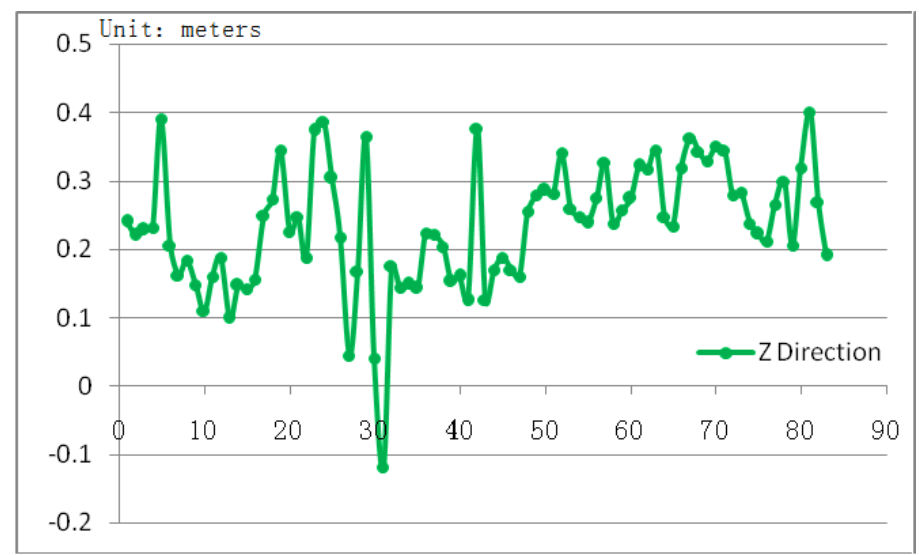

Fig. (7). The differential values of height direction.

Table 5. Laser scanner angle measurement in degrees.

\begin{tabular}{|c|c|c|c|}
\hline ID & Laser Scanner & Multi-tooth Dividing Table & Error \\
\hline 1 & 15.63 & 15.65217 & -0.02217 \\
\hline 2 & 31.23 & 31.30436 & -0.07436 \\
\hline 3 & 46.85 & 46.95653 & -0.10653 \\
\hline 4 & 62.61 & 62.60869 & 0.00131 \\
\hline 5 & 78.24 & 78.26086 & -0.02086 \\
\hline 6 & 93.83 & 93.91306 & -0.08306 \\
\hline 7 & 109.59 & 109.56522 & 0.02478 \\
\hline 8 & 125.18 & 125.21739 & -0.03739 \\
\hline 9 & 140.94 & 140.86956 & 0.07044 \\
\hline 10 & 156.4 & 156.52172 & -0.12172 \\
\hline 11 & 172.25 & 172.17389 & 0.07611 \\
\hline 12 & 187.85 & 187.82608 & 0.02392 \\
\hline
\end{tabular}


Table 5. contd...

\begin{tabular}{|c|c|c|c|}
\hline ID & Laser Scanner & Multi-tooth Dividing Table & Error \\
\hline \hline 13 & 203.44 & 203.47825 & -0.03825 \\
\hline 14 & 219.09 & 219.13042 & 0.04042 \\
\hline 15 & 234.8 & 234.78258 & -0.05478 \\
\hline 16 & 250.38 & 250.43478 & 0.06306 \\
\hline 17 & 266.15 & 266.08694 & 0.00089 \\
\hline 18 & 281.74 & 281.73911 & -0.00128 \\
\hline 19 & 297.39 & 297.39128 & -0.05347 \\
\hline 20 & 312.99 & 313.04347 & -0.08564 \\
\hline 21 & 328.61 & 328.69564 & -0.01478 \\
\hline
\end{tabular}

Angular accuracy was obtained by root-mean-square error method is $0.058^{\circ}$.

is difficult to obtain the vehicle position in real time for accuracy assessment. This problem may be solved in the future.

In this paper, the present methods were verified with the decimeter level of MLS, the methods also can assess the centimeter level of MLS because the testing field has high precision which the coordinates reach to $1 \sim 2 \mathrm{~cm}$. It has a greater error when extract the feature points coordinate for assessing the overall accuracy of MLS. The error is related to the angular resolution and scanning distance of the laser scanner, thus how to reduce extraction errors will be researched in the future.

\section{CONCLUSION}

Currently, since there are many varieties and different properties of MLSs, how to evaluate the performance of this system is particularly important. In this paper, a set of key performance evaluation methods are proposed to assess the accuracy of the sensors and the system. The methods are proved to be effective and reliable for MLS integrated accuracy assessment. The development trend of MLS in future is to improve the system by using more accurate sensors and more sophisticated technology, further expanding the scope of its application areas and applications. With the development of Chinese Smart City, the system is bound to have a great value and market demand.

\section{CONFLICT OF INTEREST}

The authors confirm that this article content has no conflict of interest.

\section{ACKNOWLEDGEMENTS}

This research is financially supported by the Surveying and Mapping Geographic Information Public Industry Scientific Research Projects under grant No.201512010.
Special thanks to Xiujuan Wu from Chinese Academy of Surveying and Mapping (CASM) for her help in improvement of the English language. The authors would also like to thank Shenghua Xu for his constructive suggestions and valuable comments.

\section{REFERENCES}

[1] K. Novak, and J. D. Bossler, "Development and application of the highway mapping system of Ohio state university," Photogrammetric Record, vol. 15, pp. 123-134, 1995.

[2] K. P. Schwarz, and N. El-Sheimy, "Kinematic multi-sensor systems for close range digital imaging," International Archives of Photogrammetry and Remote Sensing, vol. XXXI, pp.774-785, 1996.

[3] N. El-Sheimy, "A mobile multi-sensor system for GIS application in urban centers," International Archives of Photogrammetry and Remote Sensing, vol. XXXI, pp. 95-100, 1996.

[4] G. P. He, "Design of a mobile mapping system for GIS data collection," International Archives of Photogrammetry and Remote Sensing, vol. XXXI, pp. 154-159, July. 1996.

[5] G. Hunter, C. Cox, and J. Kremer, "Development of a commercial laser scanning mobile mapping system - Street Mapper," In: $2^{\text {nd }} I n$ ternational Workshop the Future of Remote Sensing, Antwerp, October 17-18, 2006.

[6] X. S. Lu, B. Shi, and D. Wang, "3DSUR-an example of the development of mobile mapping systems in China," Survey Review, vol. 43, pp. 415-426, 2011.

[7] P. Rieger, N. Studnicka, and M. Pfennigbauer, "Boresight alignment method for mobile laser scanning systems," Journal of Applied Geodesy, vol. 4, pp.13-21, 2008.

[8] R. L. Scouarnec, T. Touze, J. B. Lacambre, and N. Seube, "A new reliable boresight calibration method for mobile laser scanning applications," The International Archives of the Photogrammetry, Remote Sensing and Spatial Information Sciences, vol. XL-3/W1, pp. 67-72, 2014.

[9] C. J. Chen, H. Liu, Y. Liu, and X. Zhuo, "High accuracy calibration for vehicle-based mobile laser scanning and urban panoramic imaging and surveying system," Multispectral Image Acquisition, Processing and Analysis, vol. 8917, pp. 89170Y-1-89170Y-8, 2014.

[10] D. R. Li, Q. W. Hu, S. Guo, and Z. Chen, "Land-based mobile mapping system with its application for the Olympic games," Chinese Science Bulletin, vol. 54, pp. 2286-2293, 2009. 
[11] A. Kukko, H. Kaartinen, J. Hyyppa, and Y. Chen, "Multiplatform mobile laser scanning: usability and performance," Sensors, vol. 12, pp. 11712-11733, 2012.

[12] M. Bitenc, R. Lindenbergh, K. Khoshelham, and A. P. Van Waarden, "Evaluation of a LiDAR land-based mobile mapping system for monitoring sandy coasts," Remote Sensing, vol. 3, pp. 1472-1491, 2011.

[13] M. Rutzinger, "Tree Modelling from mobile laser scanning datasets," The Photogrammetric Record, vol. 26, pp. 361-372, 2011.

[14] H. Y. Guan, J. Li, Y. T. Yu, C. Wang, M. Chapman, and B. Yang, "Using mobile laser scanning data for automated extraction of road markings," ISPRS Journal of Photogrammetry and Remote Sensing, vol. 87, pp. 93-107, 2014.

[15] L. N. Fang, and B. S. Yang, "Automated extracting structural roads from mobile laser scanning point clouds," Acta Geodaetica et Cartographica Sinica, vol. 42, pp. 260-267, 2013.
[16] L. L. Zhu, J. H. Hyyppa, and A. Kukko, "Photorealistic building reconstruction from mobile laser scanning data," Remote Sensing, vol. 3, pp. 1406-1426, 2011.

[17] M. Vaaja, A. Kukko, H. Kaartinen, M. Kurkela, E. Kasvi, C. Flener, H. Hyyppä, J. Hyyppä, J. Järvelä, and P. Alho, "Data processing and quality evaluation of a boat-based mobile laser scanning system," Sensors, vol. 13, pp. 12497-12515, 2013.

[18] X. S. Lu, Z. Y. Zheng, D. Wang, and B. Shi, "Evaluation on theoretical accuracy of 3dsurs system scanner point," Acta Geodaetica et Cartographica Sinica, vol. 39, pp. 202-206, 2010.

[19] H. Kaartinen, J. Hyyppa, A. Kukko, A. Jaakkola, and H. Hyyppä, "Benchmarking the performance of mobile laser scanning systems using a permanent test field," Sensors, vol. 12, pp. 12814-12835, 2012.

(C) Xu et al.; Licensee Bentham Open.

This is an open access article licensed under the terms of the Creative Commons Attribution Non-Commercial License (http://creativecommons.org/licenses/bync/4.0/) which permits unrestricted, non-commercial use, distribution and reproduction in any medium, provided the work is properly cited. 